MAYO CLINIC v

\section{Evaluation of Intraoperative Fluoroscopic Techniques to Estimate Femoral Rotation: A Cadaveric Study}

David V. Ivanov'1, John P. Welby', Jonathan D. Barlow MD, MS², S. Andrew Sems MD², Michael E. Torchia MD², Brandon J. Yuan MD2

${ }^{1}$ Mayo Clinic Alix School of Medicine, Rochester, MN

${ }^{2}$ Department of Orthopedic Surgery, Mayo Clinic, Rochester, MN
Background

Intraoperative evaluation of femoral rotation (FR) utilizing fluoroscopy is an inexact science Several methods described for use during femoral nailing

Minimal data exists comparing different techniques

\section{Methods}

10 cadlavers with 20 intact femurs with premortem CT Mid-diaphyseal osteotomy created in each right femur Four surgeons used 3 techniques to match fractured femur rotation with intact contralateral side

$\mathrm{NH}$ and $\mathrm{TL}$ techniques also used by each surgeon to estimate femoral version of intact femur

Estimation comparted with CT measured rotation as "gold standard"

After obtaining a perfect lateral of the distal femur with the fluoroscopic beam perfectly parallel to the floor, the rotation of the proximal femur was assessed as described below:

\section{Neck Horizontal (NH) Angle Method}

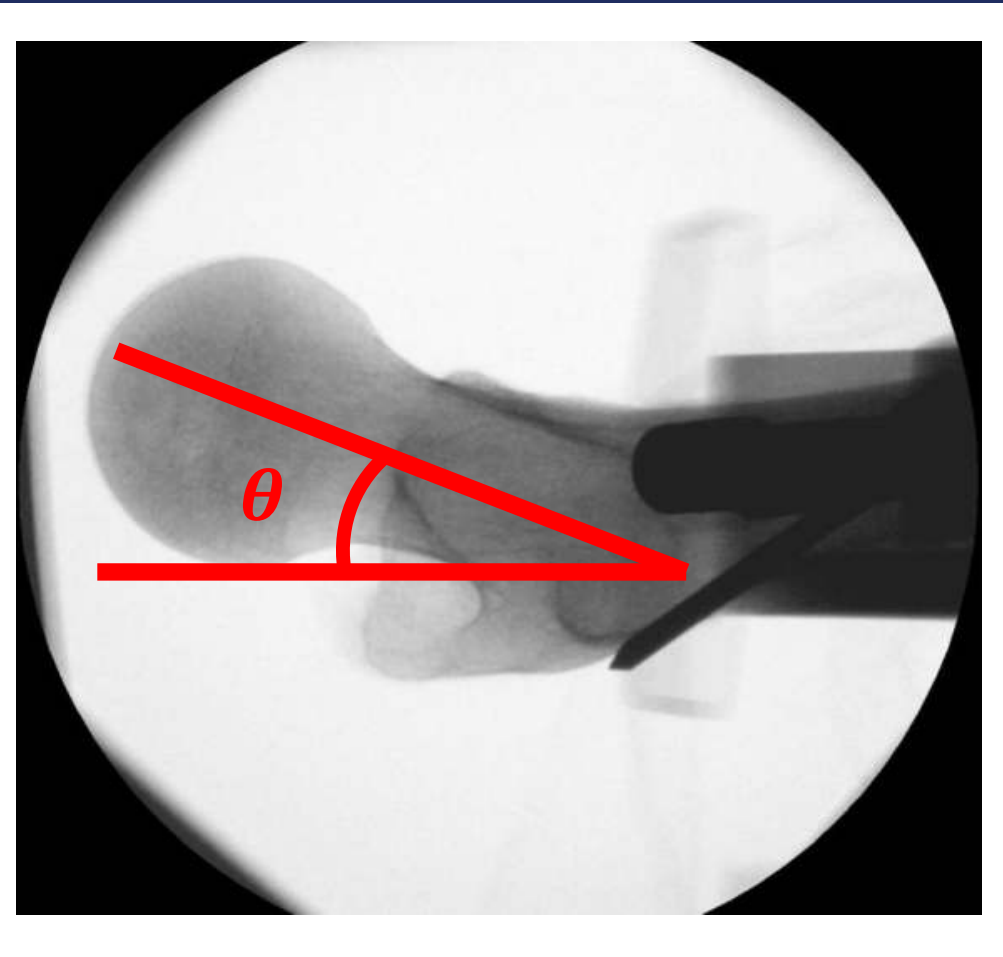

The fluoroscopic beam angle is not changed, and a lateral of the femoral neck is obtained. The angle subtended between the femoral neck and the horizontal is the estimated FT
Measured FR vs CT of Intact Femur

\begin{tabular}{|l|c|c|}
\hline & $\begin{array}{c}\text { Absolute Mean } \\
\text { Error }(95 \% \mathrm{Cl})\end{array}$ & $\begin{array}{c}\text { Interobserver } \\
\text { Agreement }(95 \% \mathrm{Cl})\end{array}$ \\
\hline NH method & $4.4^{\circ}(3.3-5.4)$ & $0.72(0.39-0.91)$ \\
\hline TL method & $8.2^{\circ}(6.5-10.0)$ & $0.69(0.27-0.91)$ \\
\hline
\end{tabular}

Measured FR of Fractured vs Intact Femur

\begin{tabular}{|l|c|c|}
\hline & $\begin{array}{c}\text { Mean Rotational } \\
\text { Error }\end{array}$ & Malrotation rate* \\
\hline NH method & $6.6^{\circ}$ & $2.5 \%$ \\
\hline TL method & $6.0^{\circ}$ & $2.5 \%$ \\
\hline LTP method & $8.5^{\circ}$ & $20 \%$ \\
\hline \hline
\end{tabular}

${ }^{*}$ Malrotation defined as rotational error $>15^{\circ}$

True Lateral (TL) Method

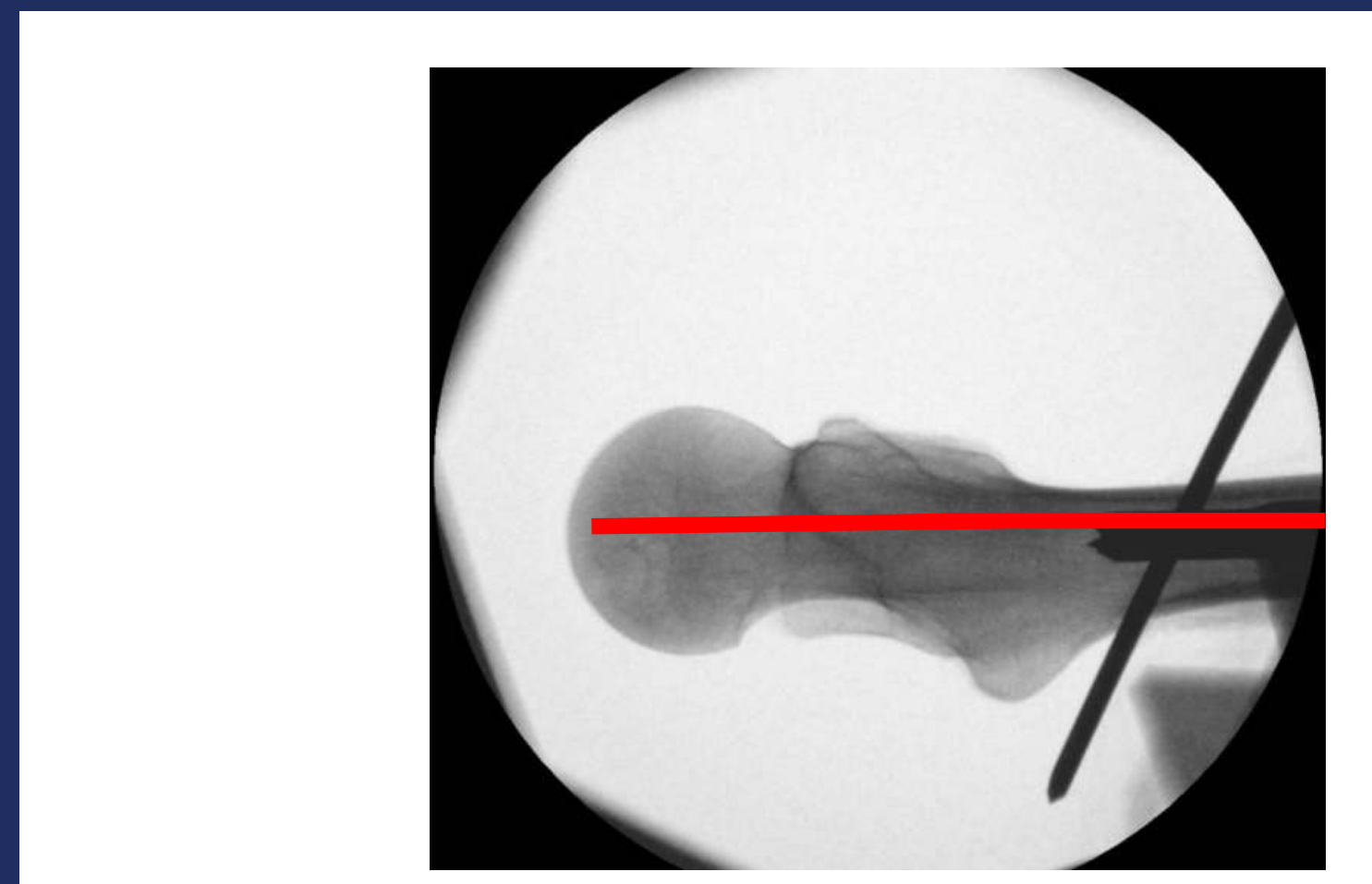

A "true" lateral of the femoral neck is obtained by rotating the fluoroscopic beam up until the image shows the femoral neck and shaft in parallel. The angle the fluoroscopic beam has rotated up to obtain this view is the estimated FT.

\section{Discussion/Conclusions}

For estimating the native FR of an intact femur:

- The NH method was more accurate vs TL method when using CT as gold standard measurement

In the fractured femur model:

- Mean rotational error was similar between all methods and within accepted clinical margin of error The LTP method malrotation rate $\rightarrow 20 \%$

- $\mathrm{NH}$ and TL methods $\rightarrow 2.5 \%$

LTP method $\rightarrow$ higher rate of malrotation and thus the $\mathrm{NH}$ and $\mathrm{TL}$ may more suitable for estimating FR during intramedullary nailing of comminuted midshaft femur fractures.

Lesser Trochanter Proffle (LTP) Method

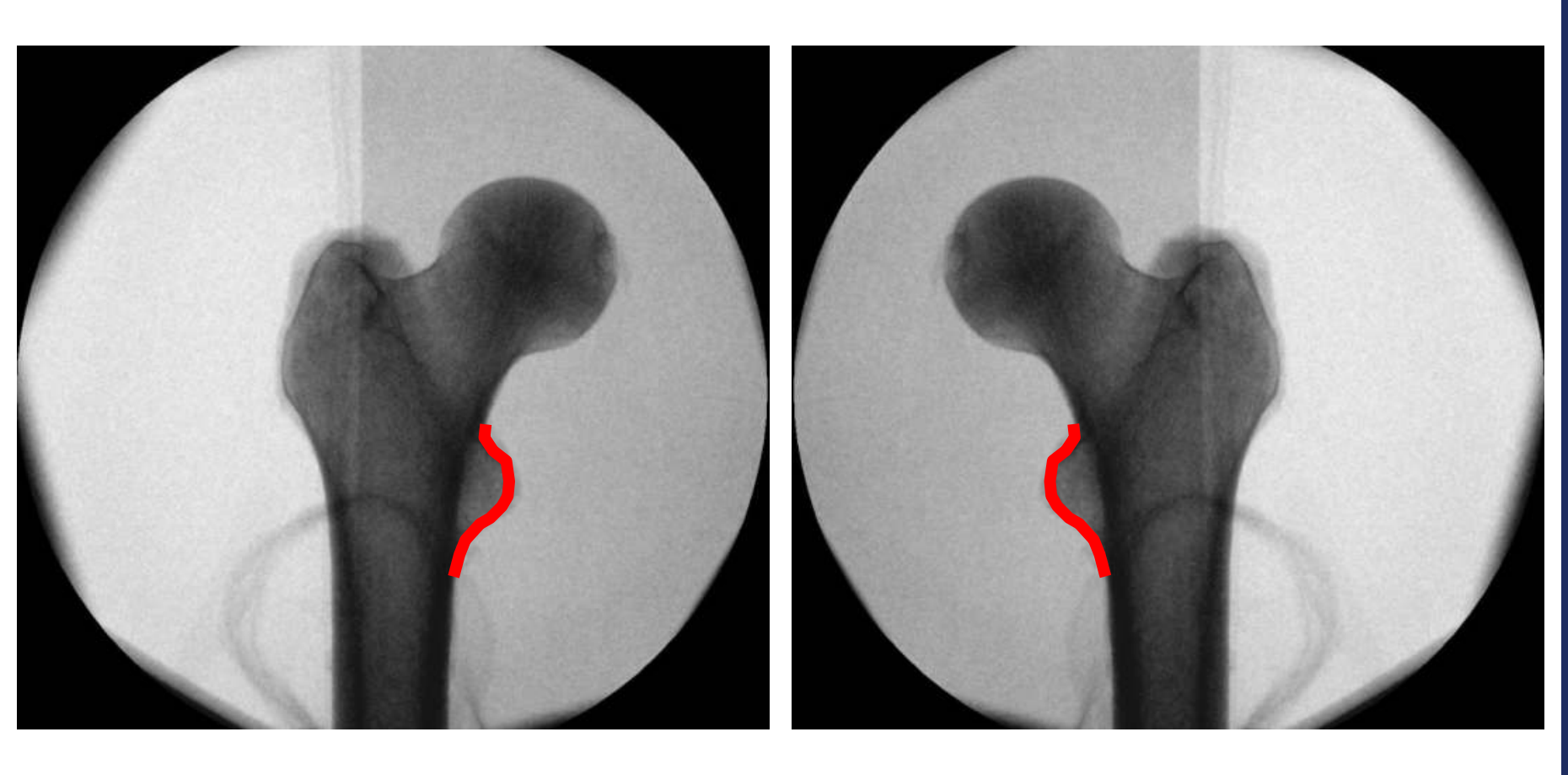

The beam is rotated 90 degrees (perpendicular to the floor) and an image of the proximal femur is obtained. The rotation of the femur is adjusted until the profile of the lesser trochanter

matches the similar image obtained from the uninjured side 